\title{
La normativa antártica de Estados Unidos y su interacción con la normativa internacional ${ }^{1}$
}

\author{
The United States Antarctic Regulation \\ and its interacction with the international law \\ Alejandra Victoria Molina MaKuc* \\ "Licenciada en Ciencias Jurídicas y Sociales de la Universidad de Chile. \\ $\bowtie$ alejandra.molinad@gmail.com
}

\begin{abstract}
RESUMEN
El presente trabajo busca caracterizar la normativa jurídica interna de Estados Unidos relativa a la Antártica. Para ello, se identifican algunos aspectos centrales que han definido la fisonomía y la evolución de su derecho doméstico y la interacción de este con las normas internacionales actualmente vigentes. En este mismo contexto de análisis se sostiene que existe en este país una regulación orgánica y sistemática relativa a la Antártica que regula las actividades de sus instituciones y ciudadanos en el territorio antártico, además de proteger los intereses de Estados Unidos en las materias antárticas. Complementariamente, se aportan antecedentes sobre cómo se ha materializado el derecho internacional antártico en este país, identificando elementos convergentes y diferenciadores entre dicho ordenamiento jurídico interno y la propia evolución de la normativa internacional sobre los asuntos antárticos.
\end{abstract}

PALABRAS CLAVE: Sistema del Tratado Antártico, Estados Unidos, Asuntos Antárticos.

\begin{abstract}
This paper seeks to characterize the United States Antarctic regulation. It identified some central aspects that have defined the physiognomy, the evolution and the interaction of the US domestic law with the international law. In this context of analysys it is held

1 Trabajo realizado a partir de Tesis para Optar al Grado de Licenciado en Ciencias Jurídicas y Sociales en la Universidad de Chile, en el marco del proyecto FONDECYT Iniciación N 11160039 (2016-2019), "Estudio comparado de la protección del medio ambiente antártico en el derecho doméstico de Chile, Argentina, Reino Unido y Estados Unidos”, cuyo investigador principal es el Dr. Luis Valentín Ferrada Walker.
\end{abstract}


that in the U.S exists an organic and systematic Antarctic regulation, that rules the activities of institutions and citizens in the Antarctic. Furthermore, preserves the U.S national interests in the Antarctic. In addition, it provides background on how Antarctic international law has materialized in the U.S, identifying convergent and differentiating elements between that domestic legal system and the evolution of the international law on Antarctic matters.

KEY WORDS: Antarctic Treaty System, United States, Antarctica.

\section{INTRODUCCIÓN}

La emergencia del Sistema de Tratado Antártico (STA), entendido como un régimen jurídico internacional que regula las actividades de distinta naturaleza que los Estados realizan en la Antártica, marcó un hito relevante en la relación de los estados nacionales con el territorio Antártico. Esta normativa, que incluye un conjunto de instrumentos internacionales, organismos y mecanismos de regulación desarrollados en el marco del Tratado Antártico de 1959, prohíbe a las partes realizar reclamaciones o derogar reclamaciones de jurisdicción en la Antártica y establece una forma de cogobierno para ella, consagrándose la Antártica como un territorio donde existen jurisdicciones concurrentes cuyo título difiere en cada caso particular (Berguño, 2009, p. 30).

De este modo, el STA distingue entre Estados reclamantes territoriales, aquellos con base de reclamación (Estados Unidos y Rusia) y países que no formularon reclamaciones antes de la firma del Tratado. Este mecanismo de cogobierno, que tiene como objetivo la protección medioambiental y la cooperación científica, requiere la cooperación internacional, coordinada a través de Reuniones Consultivas del Tratado Antártico (RCTA). Por ello, resulta de especial importancia el esfuerzo de cada una de las Partes que integran el Tratado Antártico para la mantención de este régimen y el cumplimiento de sus finalidades. Cada Parte debe realizar esfuerzos propios para asegurar que las actividades realizadas por sus ciudadanos e instituciones se desarrollen conforme a los acuerdos internacionales (Bastmeijer, 2000, p. 287).

De ahí que, resulte especialmente relevante el análisis de las legislaciones internas que cada uno de los países han desarrollado para regular sus actividades antárticas. Por ello, en este artículo se analiza particularmente el caso de Estados Unidos, considerando principalmente que dado su caracter de potencia mundial, ha desarrollado un fuerte interés antártico que lo ha llevado, no solo a transformarse en uno de los países con mayor influencia en el STA, sino que también a desarrollar una abundante regulación antártica. Es así como, las actividades que Estados Unidos realiza en la Antártica a través de sus instituciones y ciudadanos están sujetas a una combinación de regulaciones, que incluye normativas domésticas y normativas internacionales suscritas en el marco del STA. 


\section{METODOLOGÍA}

El presente trabajo busca caracterizar el derecho doméstico de Estados Unidos relativo a la Antártica, determinando algunos de los aspectos centrales que han definido la evolución de su normativa. Se persigue determinar si existe en este país una regulación orgánica y sistemática relativa a la Antártica y especificar cuales son los aspectos que se pretenden regular, proteger o normar. Del mismo modo, resulta relevante determinar cómo se ha materializado el derecho internacional antártico en este país e identificar elementos convergentes y divergentes entre dicho ordenamiento jurídico interno y la propia evolución de la normativa internacional al respecto o sobre los asuntos antárticos. La realización de este análisis comparativo, nos permitirá comprobar la hipótesis acerca de si existe o ha existido una correlación entre el desarrollo normativo interno estadounidense, sus mecanismos de generación, sus instituciones o normas más relevantes y la dinámica del desarrollo del régimen jurídico internacional que rige a la Antártica.

Desde la perspectiva de las relaciones internacionales y la cooperación internacional este análisis resulta de gran interés por cuanto Estados Unidos, a pesar de no tener reclamaciones de soberanía sobre la Antártica, se ha transformado en las últimas décadas en uno de los países con mayor actividad antártica, tanto en materia de exploración geográfica, científica, tecnológica e incluso actividad económica en las zonas reguladas en el STA, y con ello ha ido definiendo e impulsando en gran medida la agenda de los países con interés en la Antártica. Por consiguiente, el problema que se busca resolver en el marco de esta investigación se relaciona con las siguientes preguntas de investigación: ¿Cuáles son los principales mecanismos, normas e instituciones jurídicas que conforman las regulaciones domesticas en Estados Unidos? Y complementariamente: ¿Existe relación o convergencia entre la normativa doméstica estadounidense y la normativa internacional sobre la Antártica?

Desde el punto de vista metodológico, este trabajo se apoya en la revisón exhaustiva de fuentas documentales secundarias y el posterior análisis de contenido. Simultaneamente se procedió a construir macro categorías de análisis para reducir el material analizado e identificar tópicos relevantes que fueron emergiendo en el proceso de análisis. De este modo, por ejemplo, emergieron los tópicos de análisis: fines de las normativas, objetos de la regulación, entre otras categorías.

\section{Hitos en la normativa Antártica estadounidense}

La regulación de la Antártica y la determinación de su naturaleza jurídica ha sido sujeto de importantes controversias a nivel global. Ya desde principios del siglo veinte, la creciente actividad económica de tipo extractiva de recursos naturales y de exploración con fines científicos por parte de nacionales de distintos países del mundo, generó un creciente interés de diversos países por tener presencia e injerencia en este territorio, hasta el momento 
desconocido. Como señala Orrego Vicuña una vez creados estos intereses económicos, científicos y políticos, surgieron también reivindicaciones territoriales (Orrego, 1984, p. 26).

En este contexto, se inició un proceso de reclamaciones de soberanía sobre la Antártica. Dentro de los países que realizaron reclamaciones territoriales sobre el continente, se encuentra Chile en 1906 delimitada en 1940, seguido por Argentina en el mismo año, delimitada en 1942, el Reino Unido también en 1906 pero delimitada entre 1908-1917, Francia en 1912, delimitada en 1938, Nueva Zelanda reclamada y delimitada en 1923, Australia en 1933 y Noruega en 1939 (Ferrada, 2012, p. 131).

Además de estos países que realizaron reclamaciones de soberanía formales, se encuentran países como Estados Unidos y Rusia, quienes sostenían, en la época, una posición divergente respecto de la posición de los estados que reclamaban soberanía. La posición de estos países frente a la Antártica consistía en que a pesar de que no reconocían las reclamaciones de soberanía hechas por otros países, reservaron sus derechos como "potenciales reclamantes". Esta posición esgrimida por Rusia y Estados Unidos, según Orrego Vicuña (1984), fue clave en la configuración de las características y principios del posterior Tratado Antártico de 1959, que pasaría a ser el principal instrumento internacional que regula la relación de los estados con la Antártica (Orrego, 1984, p.28).

El Tratado Antártico fue suscrito en Washington, D.C., el 1 de diciembre de 1959 y entró en vigor el 23 de junio de 1961. Este instrumento fue firmado por los países que realizaron actividades científicas durante el Año Geofísico Internacional (1957-1958). Los países signatarios fueron: Argentina, Australia, Bélgica, Chile, Francia, Japón, Nueva Zelanda, Noruega, Sudáfrica, la Unión de Repúblicas Socialistas Soviéticas, el Reino Unido y los Estados Unidos de América. Los signatarios reconocieron en el Preámbulo del Tratado Antártico "que es en interés de toda la humanidad que la Antártica continúe utilizándose siempre exclusivamente para fines pacíficos y que no llegue a ser escenario u objeto de discordia internacional" (Preámbulo Tratado Antártico, párrafo 1, 1959).

Dentro de este proceso de creación de un mecanismo para el cogobierno de la Antártica, Estados Unidos ha sido un actor fundamental. Esto se puede evidenciar en distintos aspectos de la historia de la relación de esta nación con el territorio antártico. Históricamente la relación de Estados Unidos con la Antártica se remonta a finales del siglo diecinueve. En esta época exploradores, navegantes marineros, balleneros y loberos estadounidenses realizaron diversas exploraciones de esta zona y descubrieron varias de las islas antárticas. Uno de estos marineros fue Nathaniel Palmer, que el 2 de febrero de 1821 fue quien habría realizado uno de los primeros viajes de reconocimiento y exploración "conocido" en la Antártica (Joyner \& Theis, 1997, p. 21).

Complementariamente, estas expediciones inclusive tuvieron fines científicos. A modo de ejemplo, en una expedición privada, iniciada el 16 de octubre de 1829 y encabezada por Nathaniel Palmer se incluyó un grupo de científicos. Durante esta expedición, James Eights se convirtió en el primer científico en trabajar en la Antártica, específicamente en la 
South Shetland Island, lugar donde tomó muestras científicas que incluyeron los primeros descubrimientos de fósiles en este territorio (Day, 2012, p. 49).

Dentro de esta historia de expediciones privadas realizadas por estadounidenses es preciso considerar un importante hito legislativo dentro de la relación de Estados Unidos y la Antártica que ocurrió en mayo de 1836. En esta fecha el Congreso estadounidense modificó la Naval Apropiation Bill. De esta forma, a través de la dictación de esta norma se autorizó al Presidente, Andrew Jackson, a enviar una expedición a los mares del sur, expedición que fue autorizada por el Congreso en el año 1840. Esta expedición, entre otras actividades, realizó la medición de las costas de la Antártica. A pesar de ser una expedición de gran tamaño y de contar con el patrocinio del gobierno, Estados Unidos no realizó reclamaciones territoriales sobre la Antártica en esa oportunidad. Esta opción inicial sería fundamental para el desarrollo de la política antártica estadounidense en el curso del siglo veinte (Joyner \& Theis, 1997, p. 21).

Esta expedición, enviada por la marina y llamada la Expedición Wilkes en honor al Teniente Charles Wilkes, se realizó entre 1838 y 1842 y contó con financiamiento del gobierno. Debido a la extensión de la operación, que recorrió 1500 millas, Charles Wilkes se transformó en la primera persona en reconocer la existencia de un continente antártico. Esto, al lograr bordear la tierra firme de la Antártica. La Expedición Wilkes constituyó una de las expediciones más relevantes realizadas en el siglo XIX producto del gran avance científico que constituyó (Jara \& Mancilla, 2016, p. 320).

De acuerdo a lo que argumentan Joyner y Theis (1997), además de los importantes hallazgos de la Expedición Wilkes, esta excursión generó un gran interés en Estados Unidos por la Antártica (Joyner \& Theis, 1997, p.29). Sin embargo, a pesar de que a comienzos del siglo diecinueve se realizaron una serie de expediciones y de la continua actividad que realizaron marineros y balleneros dentro de los años siguientes, Estados Unidos no desarrolló un mayor interés por la Antártica, no patrocinando nuevas expediciones en este territorio. De acuerdo a Jara (2009) este bajo interés podría explicarse, en parte, por el hecho de que Estados Unidos hasta antes de la Primera Guerra Mundial, todavía no se convertía en potencia mundial (p. 2012). Otro factor a considerar es que este país, por su ubicación geográfica priorizó la exploración del Ártico. De modo que, la Antártica solo constituyó, un área de interés una vez que el Ártico había sido recorrido (Jara, 2009, p. 113).

De acuerdo a la caracterización de la presencia de Estados Unidos en la Antártica que realizan Joyner y Theis (1997) en el siglo diecinueve se inicia una etapa definida por la presencia exclusiva de expediciones privadas, no existiendo otras manifestaciones de interés de esta nación en la Antártica. Es así como, con posterioridad a la expedición de Wilkes, el siguiente explorador estadounidense que visitó la Antártica en 1929 fue el almirante Richard Evelyn Byrd. Durante los años 1928-1930 y 1933, el Almirante Byrd, fue la figura más prominente en las actividades en la Antártica de inicios del siglo veinte y quien habría "estimulado al gobierno de Estados Unidos a involucrarse activamente en la política 
Antártica” (Joyner \& Theis, 1997, p. 22). Como se ha dicho, las expediciones realizadas por Byrd no fueron financiadas con fondos públicos sino que por grandes magnates como Edsel Ford y John D. Rockefeller Jr.; contando además con el apoyo de grandes instituciones como la National Geographic Society y The New York Times (León, 2005, p. 24).

En 1926 el Almirante Byrd había logrado sobrevolar el Ártico y el deseo de ser el primer hombre en sobrevolar ambos polos lo llevó a realizar una expedición a la Antártica en 1928. Es así como, Byrd lideró dos expediciones privadas que incluyeron el primer vuelo sobre el polo sur, realizado el 28 de noviembre del año 1929. Este histórico vuelo recorrió una distancia de 1.600 millas y duró 19 horas (Jara \& Mancilla, 2016, p. 322). Además de esto, en su primera expedición, realizada entre 1928 y 1930, estableció en la Bahía Ballenas la base "Little América”. Esta base fue la más compleja establecida en la Antártica y constituyó el grupo más grande en pasar el invierno en el continente, hasta ese momento (Joyner \& Theis, 1997, p. 22). A su regreso a Estados Unidos, Byrd había adquirido una gran fama por sus descubrimientos y fue recibido como un héroe por el Presidente Franklin D. Roosevelt.

Como se observa, la construcción de Little América I en 1929 y II en 1933-1934 posicionó a Estados Unidos como el primer país en tener un establecimiento permanente en la Antártica (Jara \& Mancilla, 2016, p. 323). Esta base constituía una pequeña ciudad que incluso poseía hangares para tres aviones y antenas de comunicación de más de 18 metros (Jara, 2005, p.151). Sumado a esto Byrd en esta expedición realizó una "reclamación extraoficial" de territorio, nombrándolo "Marie Byrd Land". Como se puede observar, tal reclamación se oponía a la incipiente política antártica desarrollada por Estados Unidos hasta ese momento.

Como resultado de la reclamación realizada por Byrd, Estados Unidos se vio en la obligación de aclarar su situación jurídica respecto de la Antártica. El encargado de resolver este problema fue el Departamento de Estado, que vio una oportunidad de afianzar el liderazgo de Estados Unidos en la región. Es así como, sumado al interés del gobierno por la Antártica, surgió un creciente interés del Congreso quienes "instaron al Departamento de Estado a reconocer el potencial estratégico y económico de la Antártica” (Joyner \& Theis, 1997, p. 22). Este interés se vio reflejado en una Resolución introducida por el Senador Mullard Tydins en 1930, donde se solicitaba al presidente "reclamar todas las áreas en la Antártica que han sido descubiertas o exploradas por ciudadanos estadounidenses" (Joyner \& Theis, 1997, p. 23). Esta resolución motivó al Departamento de Estado a iniciar un estudio sobre las reclamaciones de soberanía de otros países y a analizar la política antártica existente, lo cual quedaría plasmado en un Memorándum del Departamento de Estado hecho el 22 de julio de 1930.

Este memorándum, llamado el Memorándum Boggs, fue realizado por un geógrafo del Departamento de Estado llamado Samuel Boggs. De acuerdo a lo señalado por Plott (1969) en su tesis sobre el desarrollo de la política antártica estadounidense, este documento constituiría el primer documento oficial que manifestó la posición de Estados Unidos respecto a la situación política de la Antártica. El Memorándum Boggs formulaba 
cuatro recomendaciones o "pasos previos" a la formulación por parte del Departamento de Estado de una política relativa a la Antártica. Es así como el Memorándum argumentaba la necesidad de:

[.... (1) realizar un estudio para determinar qué países tienen derecho a reclamar que zonas de la Antártica, (2) determinar, con respecto al derecho internacional y la Doctrina Hughes, el grado de ocupación efectiva requerida en las regiones polares, (3), determinar los procedimientos internos necesarios para la adquisición de territorio antártico por los Estados Unidos, y (4) determinar la actitud de los Estados Unidos con respecto a la utilización del principio del sector en la Antártica" ((Plott, 1969, p. 52).

El memorándum se refería a la doctrina articulada por Charles E. Hughes, Secretario de Estado en 1924. En este año Charles Hughes respondió a dos notas enviadas a Estados Unidos, una por el gobierno de Noruega donde se advertía la intención de reclamar soberanía sobre los territorios descubiertos durante el viaje aéreo transártico de Roald Amundsen y la otra por la Republican Publicity Association que consultaba si es que existía una reclamación de soberanía sobre las tierras descubiertas por Wilkes en la expedición de 1840. En su respuesta a estas comunicaciones Hughes argumentó que el solo descubrimiento, sin un asentamiento, no proveía a un país de una reclamación legítima de soberanía. Esta estricta interpretación, implicaba que un país debía instalarse, colonizar y explotar un territorio para ser considerado como legítimo reclamante. En oposición a esta doctrina, el año siguiente el Ministro del Interior de Canadá, Charles Stewart, sostuvo que Canadá reclamaba soberanía sobre un "sector" que comprendía todos los territorios dentro de su línea costera norte hasta el polo norte. Este posición adoptada constituye el llamado "principio del sector", que a diferencia de lo planteado por la Doctrina Hughes no requería una ocupación efectiva de los territorios (Lackenbauer \& Kikkert, 2015, p.220). La doctrina Hughes era parte del Nuevo Imperialismo de fines del siglo diecinueve y comienzos del siglo veinte. Lo que buscaba Estados Unidos con la implementación de esta política era establecer un orden internacional que le permitiera explotar su creciente poder económico. A pesar de que la Doctrina no se refería específicamente a la Antártica, esta se extendió a ella. Es así como Estados Unidos, para preservar su posibilidad de acceder a este territorio, no reconoció las reclamaciones de otros países (Hall, 1989, p. 137). En este sentido, la doctrina Hughes le permitió a Estados Unidos no definir su posición frente al Ártico y la Antártica, no realizando reclamaciones de soberanía pero conservando su derecho a hacerlo en el futuro (Lackenbauer \& Kikkert, 2015, p.220).

Por su parte, el Memorándum Boggs concluía que Estados Unidos debía optar entre realizar una reclamación territorial o promover la creación de un acuerdo internacional Las recomendaciones hechas por Boggs tienen una importancia histórica y jurídica, puesto que reflejan la posición que Estados Unidos adoptaría en el futuro, respecto de la Antártica. 
Asimismo, como señalamos constituye uno de los primeros documentos oficiales que manifiesta la posición y los intereses geopolíticos del país sobre la Antártica.

En el año 1939 se inició una segunda etapa en la relación de Estados Unidos con la Antártica caracterizada por la presencia de expediciones financiadas por el gobierno estadounidense. La política antártica estadounidense a fines de la década de 1930, sustentada en la doctrina Hughes, era no reconocer ninguna reclamación extranjera, pero conservando el derechos que pudiesen tener en el futuro. En contraposición, se encontraba la propuesta de Boggs que sugería que los descubrimientos de Byrd no contravenían las reclamaciones de otros países y que en razón de la potencialidad económica de la zona era recomendable una reclamación. Por su parte, el Departamento de Estado en 1938 tenía una política no oficial que promovía la conveniencia de presentar reclamaciones individuales sobre territorios inexplorados. Lo que se observa en las recomendaciones que realizó el Secretario Cornell Hull al explorador Ellsworth respecto a los beneficios de que las reclamaciones individuales podrían tener. A pesar de esta situación, en 1940 el Subsecretario Sumner Welles incentivó la tesis de Hughes, que exigía ocupación efectiva. Es en base a esta tesis que se realizó la expedición del United States Antarctic Service (León, 2005, p. 68).

En los años 1939 y 1940 se realizaron las expediciones del United States Antarctic Service bajo el liderazgo de la Marina de Estados Unidos pero con una fuerte presencia de civiles encargados de los objetivos científicos de las expediciones. Producto de que en esta época diversos países comenzaron a realizar reclamaciones territoriales y que exploradores de la Alemania Nazi llegaron a la Antártica, el interés de Estados Unidos creció en relación a establecer regulaciones para el acceso al continente blanco (Joyner \& Theis, 1997, p. 25).

Estos antecedentes serían lo que explicaría que el gobierno de Franklin D. Roosevelt implementara una "política antártica más agresiva", que se habría basado en las recomendaciones hechas por el Memorándum Boggs. Esta postura se puede evidenciar en un discurso pronunciado en el año 1939, por el presidente Franklin D Roosevelt, quien declaró al Oficial al mando del U.S Antarctic Service:

[....] los Estados Unidos nunca han reconocido las reclamaciones de soberanía sobre el territorio en la región antártica afirmada por cualquier estado extranjero. Ningún miembro del United States Antarctic Service deberá tomar cualquier acción o declaraciones tendientes a comprometer esta posición" (Abdel- Motaal, 2016, p.55).

A partir de este discurso, se puede observar cómo se instala la noción de soberanía que sostenía Estados Unidos sobre la Antártica. Esta postura evolucionaría en el tiempo y pasaría a convertirse en la base de su propuestas a la comunidad internacional.

En efecto, esta postura revela lo que sería la futura propuesta de Estados Unidos frente a las reclamaciones de otros países sobre la Antártica. De esta manera, en el transcurso del mismo año 1939 el Presidente Roosevelt autorizó una expedición oficial a 
la Antártica. El Congreso, pese a su reticencia inicial por los costos asociados, autorizó los fondos necesarios para la expedición gubernamental, encabezada por el Almirante Byrd. Estas operaciones constituían el U.S Antarctic Service, que mantuvo las bases de Marguerite Bay y Bay of Whales. Como explican Joyner y Theis (1997) la creación del U.S Antarctic Service, responde a un interés estratégico y de carácter geopolítico de Estados Unidos de establecer una presencia permanente en la Antártica. En esta línea, el 25 de noviembre de 1939 el Presidente Roosevelt firmó la directiva orgánica que creó al US Antarctic Service como una Agencia de Gobierno parte del Departamento de Interior. Sin embargo, a pesar del creciente rol que estaba adquiriendo esta Agencia, la Segunda Guerra Mundial, tuvo como consecuencia una disminución de su presupuesto, lo que finalmente llevó a la evacuación de las dos bases en 1941(Joyner \& Theis,1997, p. 25).

Una vez finalizada la Segunda Guerra Mundial, las actividades de Estados Unidos aumentaron exponencialmente. Evidencia de esto es que entre 1946 y 1947 este país realizó la operación High Jump que fue la exploración más grande realizada por Estados Unidos en la época, involucrando a más de 4,700 personas. En esta expedición se habrían realizado, entre otras actividades, la toma de fotografías aéreas de la Antártica y habría tenido como objetivo principal "entrenar a miembros de la armada y probar naves, aeronaves y otros equipos militares bajos las condiciones del frío glacial”(Saul \& Stephens, 2015, p. 34). Los objetivos detrás de esta expedición podrían encontrarse en que uno de los fines perseguidos por Estados Unidos en la época de la posguerra fue la búsqueda de prestigio en la arena internacional. En este sentido, la Antártica ofrecía una oportunidad para mostrar su liderazgo, puesto que dos grandes potencias realizaban importantes actividades en la Antártica, estas eran Gran Bretaña y la Unión Soviética, quien por su búsqueda de supremacía representaba una amenaza para Estados Unidos (León, 2005, p. 39). Es así como, el deseo de reconocimiento de su liderazgo lo llevó a organizar la Operación High Jump. Como uno de los objetivos de la operación era circunnavegar la Antártica, se especuló que la operación High Јитр creaba un sustento para reclamar los territorios explorados. En este sentido el Almirante Byrd y el Departamento de Estado creían que la operación creaba una base para una reclamación e instaron al Gobierno a reevaluar la Doctrina Hughes de 1924(Moore, 2005, p. 197). De manera que, entre 1944 y 1948 la Marina de Estados Unidos se convirtió en la agencia dominante a través de las operaciones High Jump (1946-1947) y Windmill (19471948) (Dodds, 2009, p. 32).

La guerra fría definiría una nueva etapa dentro de la relación de Estados Unidos con la Antártica. Los ciudadanos rusos desde el siglo diecinueve habían realizado actividades de navegación y balleneras en la Antártica, que continuaron durante el siglo veinte. Esta situación en un contexto de Guerra Fría amenazaba claramente la posición de Estados Unidos respecto de la Antártica. En este sentido, Mancilla (2005) argumenta que esta situación motivó a Estados Unidos a comenzar a realizar negociaciones para impedir que la Unión Soviética realizara alguna reclamación territorial que pudiera afectar sus intereses 
geopolíticos (p. 84). En este contexto de constantes disputas entre Estados Unidos y la Unión Soviética, la Antártica se convirtió en un "atractivo espacio” para el desarrollo de operaciones militares por parte de los estadounidenses. De acuerdo a Mancilla (2005) las intenciones de Estados Unidos de evitar una reclamación soviética se aprecian, claramente, en 1948 cuando el gobierno de Estados Unidos realizó un "proceso de consulta a las naciones que tenían intereses en la región Antártica para llevar a cabo un plan de fideicomiso y posteriormente un proyecto de internacionalización" (p. 84). Estas propuestas son los primeros antecedentes de una preocupación del gobierno estadounidense de regular sus actividades y las del resto de la comunidad internacional en la Antártica.

\section{Normativa Antártica Internacional: Aspectos Históricos y Jurídicos relevantes}

A partir de la segunda postguerra mundial y en el contexto de la Guerra Fría el interés de Estados Unidos en la Antártica creció notablemente (Diáz \& Villamizar, 2014, p. 17-31). Este interés se vio también influenciado por las demarcaciones de los territorios reclamados de Chile (1940) y Argentina (1942) que evidenció que los territorios antárticos chileno, argentino y británico se superponían, generando un aumento de la tensión en la zona. Entre 1947 y1953 Chile intensificó sus actividades en la Antártica, con la instalación de tres bases, entre las que se encuentra la Base Bernardo O’Higgins en 1948 y con el envío de flotas navales. En este contexto, Gran Bretaña en 1948 envió una nota de protesta a Argentina y Chile por las actividades que estos países estaban realizando, sugiriendo la posibilidad de realizar un arbitraje de la Corte Internacional de Justicia de La Haya para dirimir el asunto (Mancilla, 2006, s/p). De esta forma, las actividades que realizaron Argentina y Chile contribuyeron a una agudización del "problema Antártico" que posteriormente llevaría a que el Reino Unido presentará dos demandas ante la Corte Internacional de Justicia el 4 de mayo de 1955 contra Chile y Argentina. Dichas demandas pretendían demostrar que ambos Estados ocupaban ilegítimamente el territorio reclamado por Reino Unido, constituyendo una violación de su soberanía (Ferrada, 2015, p. 158).

Es en este contexto, que Estados Unidos buscó resolver los potenciales conflictos en la zona y promovió la creación de una solución internacional para el status político-legal de la Antártica. Todo esto con el objetivo de normalizar las relaciones entre Reino Unido, su histórico aliado internacional y las naciones sudamericanas con presencia antártica, a través de diversas y sucesivas propuestas de regulación internacional del territorio antártico. Estas propuestas además podrían tener un objetivo paralelo: fortalecer la propia presencia de Estados Unidos en la Antártica, debilitando las posiciones de Chile, Argentina y Reino Unido (Llanos, 2008, p. 178).

Una de las primeras propuestas fue presentada por el Departamento de Estado estadounidense en 1947 (Joyner \& Theis, 1997, p. 74). En esta ocasión se propuso como solución al problema de la Antártica la creación de un fideicomiso internacional por parte de 
las Organización de las Naciones Unidas (ONU). Esta propuesta se basaba en lo dispuesto por los artículos 75, 76, 77 y 79 (Capítulo XII) de la Carta de Naciones Unidas (1945) que consagran la posibilidad de creación de un régimen internacional de administración fiduciaria para ciertos territorios. Sin embargo, esta solución adolecía de un problema de aplicabilidad por tratarse de uno de los territorios más despoblados del planeta (Villamizar, Ibarra \& Guerrero, 2013, p. 110).

Ante este problema de aplicabilidad, el Departamento de Estado planteó una segunda propuesta: la creación de un "condominio" (Departamento de Estado, 2017). Esta idea consideraba que la Antártica quedara "bajo la autoridad y control directo de los Estados interesados”. De igual modo, este condominio consideraba a la Organización de Naciones Unidas como colaboradora y excluía a la Unión Soviética de la administración del territorio . Por su parte, la propuesta de internacionalización buscaba potenciar la investigación científica de los países con interés en la Antártica y el mantenimiento del status quo de las reclamaciones de los países para delegar a un gobierno común (Mancilla, 2004, p. 162). De esta forma los dos principios que regirían el condominio serían: la desmilitarización del territorio antártico y la libertad de exploración e investigación científica (Diaz \& Villamizar, 2014, p. 17-31). Esta propuesta fue presentada a los países que habían realizado reclamaciones o delimitaciones en la Antártica, estos eran: Chile, Argentina, Gran Bretaña, Noruega, Australia, Francia y Nueva Zelanda y negociada en los años siguientes, sin embargo, pese a la novedad de la propuesta, esta no tuvo mayor acogida en la comunidad internacional (Mancilla, 2004, p.158).

Como se observa de lo señalado anteriormente, el Departamento de Estado tuvo un importante rol en la época. Además de ser los encargados de redactar las propuestas de una solución jurídica para la Antártica, apoyaron los esfuerzos científicos para planificación del AGI que se desarrollaría entre el 1 de julio de 1957 y el 31 de diciembre de 1958.

\section{Antecedentes políticos y jurídicos del Tratado Antártico}

Los años 1957 y 1958 fueron esenciales tanto en el desarrollo de la normativa antártica estadounidense como para la normativa internacional. Durante estos años se celebró el Año Geofísico Internacional (AGI), el cual promovió la realización de expediciones polares e incluyó la realización de investigaciones por parte de doce países en 67 estaciones en la Antártica. A pesar de la importancia del AGI en la consagración de los principios de la cooperación internacional antártica, estos tuvieron como antecedente la Segunda Conferencia Preparatoria (Berna, 1880) del Primer Año Polar Internacional (1882- 1883) donde se desarrolló la idea de la cooperación (Berguño, 2009, p.25).

La realización de un Año Geofísico Internacional enfocado en la Antártica se debe a la iniciativa de un grupo de investigadores geofísicos que en 1950 comienzan a promover la realización de un Tercer Año Polar para realizar estudios geofísicos sobre la Tierra 
(Buedler, 1957, p. 20). En octubre de 1951 dicha idea se reforzó cuando el Comité Ejecutivo del Consejo Internacional de Uniones Científicas (CIUS), formado en 1931 y cuya misión era apoyar el desarrollo científico mundial, aprobó la solicitud de estos investigadores que buscaban estudiar una serie de fenómenos geofísicos desde altas latitudes (Aguayo, 2005, p.31). Posteriormente, la Unión Internacional de Geodesia y Geofísica se reunió en Roma en 1954 para crear un Comité Especial encargado de establecer un plan para determinar la ubicación de las bases de investigación en la Antártica. En 1955 el Comité Especial para el AGI se reunió en París, contando con la presencia de representantes de: Argentina, Australia, Bélgica, Estados Unidos, Francia, Gran Bretaña, Japón, Noruega, Nueva Zelanda, Suecia y la Unión Soviética (Pinochet de la Barra, 1976, p. 171). En estas reuniones de planificación participaron los siete estados reclamantes, más Estados Unidos. Bélgica y la Unión Soviética. En esta oportunidad se fijaron las áreas y bases donde se desarrollarían las actividades científicas (Ferrada, 2014, p. 9).

Dentro de la literatura se ha vinculado la celebración del Año Geofísico Internacional y con el desarrollo del Tratado Antártico. Sin embargo, esta vinculación buscaría principalmente justificar la creación del Tratado como un mecanismo motivado por la ciencia y la protección del medioambient, desconociendo, sus justificaciones políticas y territoriales (Ferrada, 2012, p.7).

A pesar de estas consideraciones el AGI fue un hito en la historia Antártica, pues permitió crear interés en las distintas naciones sobre las potencialidades y el valor científico que esta poseía. El AGI se desarrolló entre el 1 de julio de 1957 y el 31 de diciembre de 1958, periodo en el cual se realizaron importantes descubrimientos científicos: se encontraron cadenas de montañas y se realizaron estudios sobre los hielos y depósitos de minerales. Estados Unidos, estableció siete estaciones en la Antártica: 1) Little América; 2) Hallet; 3) Wilkes; 4) Ellsworth; 5) Byrd; 6) South Pole y 7)Mc Murdo Sound (United States Antarctic Program, 2014). El establecimiento de estas bases permitiría fortalecer las actividades científicas y de exploración realizadas por el país.

Dentro de la creación y el desarrollo de un marco regulatorio de las actividades realizadas por Estados Unidos en la Antártica, es preciso considerar la creación del United States Antarctic Program en 1959. Este programa se centró en el desarrollo de distintas actividades científicas, como el mapeo, estudios de la biología, océanos, la geología, atmósfera y glaciología. El año 1953 la National Academy of Sciences estableció el U.S. National Committee,que fue el encargado de organizar la participación de Estados Unidos en el AGI (National Academy of Sciences, 2017). Por otra parte, a la National Science Foundation (NSF), una agencia federal establecida en 1950, se le encomendó la facultad de administrar el financiamiento otorgado por el Congreso para los proyectos científicos.

Pese a que el AGI estuvo marcado por un contexto de guerra fría, la organización de este evento, logró crear un "ambiente propicio” para suscribir el Tratado Antártico (Ferrada, 2012, p. 284). De esta forma, en el año 1958, el presidente Dwight D. Eisenhower invitó a las 
otras 11 naciones participantes del Año Geofísico Internacional a una reunión en Washington D.C. para negociar lo que concluiría siendo el Tratado Antártico. En esta ocasión Estados Unidos manifestó cuáles eran sus intereses en la Antártica y su posición política al respecto. En este sentido las palabras del secretario de Estado John Foster Dulles ilustran claramente la posición de Estados Unidos:

[....] en vista de las actividades de los Estados Unidos y sus ciudadanos mencionados, mi Gobierno se reserva todos los derechos de los Estados Unidos con respecto a la región antártica, incluyendo el derecho a hacer valer una reclamación o reclamaciones territoriales [...] Es la opinión de mi Gobierno, sin embargo, que los intereses de la humanidad serían mejor cumplidos, en consonancia con los altos ideales de la Carta de las Naciones Unidas, si los países que tienen un interés directo en la Antártida se unieran en la conclusión de un tratado que tendría los siguientes fines pacíficos: A. La libertad de investigación científica en toda la Antártica por ciudadanos, organizaciones y gobiernos de todos los países B. Acuerdos internacionales para garantizar que la Antártica se utilizará exclusivamente para fines pacíficos. C. Cualquier otro fin pacífico no incompatible con la Carta de las Naciones Unidas. Se cree que un tratado de esa índole puede concluirse sin requerir que ninguna nación participante renuncie a cualquier derecho histórico básico que puede tener en la Antártica, o cualesquiera reclamaciones de soberanía pueda haber afirmado. Podría ser expresamente dispuesto que dichos derechos fundamentales y tales reivindicaciones no se verían afectadas, mientras el tratado este en vigor, y que nuevos derechos no serían adquiridos y nuevas reclamaciones no serían hechas por ningún país durante la duración del tratado" (U.S. Antarctic Program, 2017, p. 2)

Esta nota, emitida en 1958, refleja la posición de Estados Unidos frente a Antártica, la cual se vería plasmada posteriormente en el Tratado Antártico. Lo expuesto, evidencia la evolución que tuvo la relación de Estados Unidos con la Antártica. Asimismo, muestra como esta relación estuvo marcada fuertemente por los intereses geopolíticos, económicos y científicos que el país tenía en la Antártica y en el hemisferio sur. En diciembre de 1959 los doce estados firmaron el Tratado Antártico: Argentina, Australia, Bélgica, Chile, Estados Unidos Francia, Japón, Noruega, Nueva Zelanda, Reino Unido, Sudáfrica y la Unión Soviética.

Es importante entender las negociaciones del Tratado Antártico en el contexto mundial de la posguerra y Guerra Fría. Puesto que, es en este contexto que Estados Unidos desarrolló su interés antártico. De esta forma, inclusive el Departamento de Estado en julio de 1959, reconocía que "la ciencia antártica era el "escudo" de la superpotencia autoproclamada como defensora del "mundo libre" (Ferrada, 2012, p. 6). Es así como el desarrollo de la ciencia fue utilizado como una herramienta para la protección del interés nacional de Estados Unidos.

En este sentido, la Antártica a lo largo del siglo veinte pasó a constituirse en un área de gran interés para Estados Unidos. Como se observa, los intereses de Estados Unidos desde 
un comienzo fueron económicos, geopolíticos y científicos. Es por este motivo que su política internacional se basó, desde un comienzo, en la búsqueda de una solución pacífica para la Antártica. Como se mencionó, las motivaciones detrás de estas propuestas estarían la necesidad de evitar un conflicto armado y de consolidar la posición de este país en la región.

Como se ha dicho Estados Unidos fue un activo promotor de propuestas y búsqueda de soluciones diplomáticas. A pesar de que este país reclama que su relación con la Antártica se remonta a inicios del siglo diecinueve, es en el siglo veinte cuando demuestra un significativo interés en la zona. De esta forma, en esta época se construye la política antártica del país y se busca el posicionamiento de su postura dentro de la comunidad internacional. Esta posición sería, en una primera instancia, el no reconocimiento de las reclamaciones de soberanía de otros países y la reserva de reclamaciones futuras. Y en una segunda instancia, la promoción de la creación de un sistema de administración internacional.

Como se puede observar, el comienzo del siglo veinte se caracterizó por el nacimiento del interés de Estados Unidos por la Antártica y por la formulación de una política antártica propia. Producto de la incipiente política antártica desarrollada en la época y la falta de claridad sobre la situación jurídica de la Antártica, el gobierno no se enfocó en el desarrollo de una regulación antártica propia. No es sino hasta después de la segunda mitad del siglo veinte cuando se inicia la creación de un marco regulatorio. Es en este contexto, que se dictan una serie de normativas, en su mayoría vinculadas con las normativas internacionales suscritas por este país.

\section{Rol de Estados Unidos en el Sistema del Tratado Antártico} y capacidades logísticas diferenciadas en el territorio

En relación a la capacidad logística de los países en la Antártica, un buen predictor de ella es la cantidad de investigaciones publicadas y el presupuesto para la realización de actividades científicas y tecnológicas son una muestra global del rol que tiene cada una de las Partes Consultivas en el Sistema del Tratado Antártico. Actualmente, Estados Unidos, opera tres bases: McMurdo Station, Amundsen-Scott South Pole Station y Palmer Station. Lo que, de acuerdo a la investigación de Andrew D. Gray y Kevin A. Hughes lo trasforma en el país con mayor presencia poblacional en la Antártica y mayor investigación científica, contando con 4.485 artículos publicados, seguido por Reino Unido, Australia y Alemania. Por su parte, Dastidar, concluye que 19 de los 20 países con mayor producción son Partes Consultivas de Tratado Antártico y los top 20 incluyen a todos los firmantes originales del tratado.

Asimismo, Estados Unidos es uno de los que países que más invierte en investigación científica. De un presupuesto total de la NSF de más de US\$7 mil billones de dólares, alrededor de US\$350 millones se destinan para el United States Antarctic Program. Lo siguen, Alemania, Reino Unido, Australia y Japón. A pesar de estos datos, en un informe elaborado en 2012 por el United States Antarctic Program Blue Ribbon Panel, se concluyó que era necesario aumentar 
el presupuesto y adquirir nuevos rompehielos para asegurar la capacidad de investigación y la presencia en el polo sur. En contraposición, China ha emergido en el escenario estableciendo a la antártica como prioridad nacional estratégica. Actualmente, China tiene cuatro bases permanentes y aumentó su productividad científica entre 1992 y 2010. Se puede concluir que, de los 12 países firmantes del Tratado Antártico, los siete reclamantes, más Estados Unidos y Rusia, no solo desarrollan la mayor cantidad de investigación científica, sino que, además, tienen la mayor influencia política en el STA. A pesar de este liderazgo, la capacidad logística de E.E.U.U está basada en inversiones realizadas durante la Guerra Fría, que no serían suficientes en el actual escenario mundial.

\section{Normativa doméstica estadounidense dictada autónomamente}

La regulación de las actividades de Estados Unidos en la Antártica se ha visto fuertemente influenciada por la normativa internacional. Dentro de los instrumentos que forman parte del Sistema del Tratado Antártico se encuentran convenciones, medidas, recomendaciones y resoluciones acordadas a través del mecanismo de Reuniones Consultivas. Como las disposiciones del Tratado Antártico son genéricas, el mecanismo de las Reuniones Consultivas ha contribuido a la ampliación de los alcances de lo dispuesto en el Tratado.

Es así como, a través de la celebración de Reuniones Consultivas, de las discusiones asociadas a estas reuniones y de la adopción de recomendaciones y convenciones, la regulación Antártica ha evolucionado. En este sentido, el Tratado Antártico dispone en su artículo X: "Cada una de las Partes Contratantes se compromete a hacer los esfuerzos apropiados, compatibles con la Carta de las Naciones Unidas, con el fin de que nadie lleve a cabo en la Antártica ninguna actividad contraria a los propósitos y principios del presente Tratado".

De ahí se desprende que es deber de los estados adoptar su derecho interno a las normativas y acuerdos internacionales que suscriban y otorgarles, de esta forma efectos jurídicos dentro de su normativa doméstica. Estas ideas fueron ratificadas en el artículo XIII del Protocolo al Tratado Antártico sobre Protección del Medio Ambiente (1991) que prescribe: "Cada Parte tomará medidas adecuadas en el ámbito de su competencia para asegurar el cumplimiento de este Protocolo, incluyendo la adopción de leyes y reglamentos, actos administrativos y medidas coercitivas". Asimismo, la norma dispone que cada Parte notificará a las demás las medidas que adopte para asegurar el cumplimiento y los esfuerzos para no realizar actividades contrarias a los propósitos del Protocolo. De ahí que Estados Unidos ha cumplido esta obligación a través de la creación de un marco legal que incluye leyes, reglamentos y permisos administrativos dictados por agencias federales.

El derecho estadounidense ha optado por regular de diversas formas su relación con la Antártica. Por ejemplo, en algunos casos, puede aplicarse extraterritorialmente la ley de Estados Unidos en la Antártica. Este es el caso de la Antarctic Conservation Act (Antarctic Conservation Act of 1978. Pub. L. 95-541, 92 Stat. 2048, 16 U.S.C. \$§ 2401 et seq), norma 
que establece penas civiles y criminales por una serie de actividades prohibidas. De acuerdo a esta normativa la NSF y el Departamento de Justicia son los responsables de hacer cumplir lo dispuesto por la ley. Sin embargo, la regulación estadounidense no es uniforme y varía dependiendo del asunto que se busca regular y la existencia o no de instrumentos internacionales. De esta forma, se buscará determinar cuáles han sido las formas de regulación de las actividades de Estados Unidos en la Antártica y cuál ha sido la vinculación entre la normativa internacional y el desarrollo su normativa doméstica.

Debido al importante rol que ha tenido Estados Unidos en el desarrollo de la normativa internacional antártica es preciso, al realizar este análisis, distinguir entre la regulación dictada autónomamente y la dictada en aplicación de acuerdos internacionales. A pesar de que, la regulación domestica estadounidense se ha visto fuertemente influenciada por la normativa internacional, existen ciertas materias que han sido desarrolladas con prescindencia de esta.

\section{Normativa Antártica Interna Estadounidense}

y su interacción con las normas del derecho internacional antártico

Esta sección está organizada sobre la base de la siguiente pregunta orientadora: ¿Cuál es la relación que existe entre el derecho doméstico estadounidense y la normativa internacional antártica? En este contexto, se analizan las vinculaciones existentes entre la normativa interna estadounidense y la normativa internacional. Para facilitar este análisis comparativo se elaboró un esquema conceptual que reúne un conjunto de criterios de comparación entre ambas normativas con el objetivo de establecer similitudes, diferencias y aspectos singulares de la normativa antártica doméstica respecto a la normativa internacional.

En Estados Unidos existen una serie de leyes que regulan las actividades de sus instituciones y ciudadanos en la Antártica. En un primer nivel de análisis se ha identificado y caracterizado dos grupos de normas antárticas estadounidenses. Un primer grupo está constituido por aquellas leyes que han sido dictadas de forma previa a que Estados Unidos suscriba acuerdos internacionales para regular esta materia (Marine Mammal Protection Act, la Antarctic Conservation Act de 1990). Un segundo grupo de normas, está conformada por aquellas disposiciones que Estados Unidos ha dictado domésticamente, pero con la finalidad de implementar acuerdos internacionales suscritos en el marco del Sistema del Tratado Antártico.

Del análisis y la recopilación de las normas, se aprecia que existe una gran influencia de Estados Unidos en los procesos de negociación y suscripción de acuerdos internacionales y a su vez que existe una tendencia de Estados Unidos de implementar autónomamente los acuerdos internacionales suscritos por este país, a través de la dictación de leyes específicas. Al respecto, cabe destacar que en Estados Unidos el derecho internacional se ha materializado a través de la dictación de leyes y reglamentos para las agencias federales que tienen responsabilidades de fiscalización de las actividades desarrolladas por sus ciudadanos en la Antártica. 
Para la aplicación del derecho internacional es necesaria la incorporación de este derecho a la normativa interna través de la dictación de normativas internas. Esto porque a persar de que el derecho internacional regula cuestiones que si bien han sido suscritas voluntariamente por los estados y estos tienen el deber de incorporarlas, formando parte de sus normativas nacionales, los estados tienen autonomía en lo relativo a la forma de implementación. En el caso de la Antártica la normativa internacional en materia recursos naturales y medidas de protección al medioambiente se materializa, cuando en los ordenamientos nacionales se dictan normativas para hacer cumplir las disposiciones internacionales (Bastmeijer, 2000, 287). De esta forma, su aplicación es competencia de los órganos judiciales nacionales, funcionarios y agencias federales. Entre estos órganos los más relevantes en Estados Unidos son: el Departamento de Estado, la National Science Foundation, National Oceanic and Atmospheric Administration y la Environmental Protection Agency.

Considerando lo anterior, el impacto del derecho internacional en el desarrollo del ordenamiento antártico interno es mayor, dependiendo del tipo o naturaleza de las regulaciones acordadas. En el caso de Estados Unidos en general el desarrollo del derecho internacional no ha ido acompañado de una consolidación en el ordenamiento interno, sino que los tribunales superiores estadounidenses, han sostenido la supremacía constitucional y la independencia de las instancias nacionales respecto a las obligaciones derivadas del derecho internacional. Esto, sin embargo, no se observa en el caso específico de la normativa Antártica (Trius, 2016, p. 178).

Como una forma de profundizar en la comparación de ambas legislaciones se han seleccionado los siguientes tópicos de comparación: i) fines de las diferentes normas antárticas; ii) jurisdicción criminal; iii) regulación de recursos marinos; iv) regulación de la exploración y explotación de recursos minerales existentes en la antártica; y v) normas de protección del medio ambiente. El desarrollo de cada uno de estas líneas de comparación se presenta a continuación.

\section{Fines}

Una primera diferencia que se puede observar es que los propósitos de ambos ordenamientos son diferentes. En el caso de Estados Unidos el interés de la creación de un conjunto de normas que regule sus actividades en la Antártica obedece a una decisión de política internacional que tiene como principal propósito la mantención de la estabilidad política en la zona, evitando conflictos entre las partes reclamantes. Esto, porque algunos de los países reclamantes son sus aliados históricos, como es el caso de Gran Bretaña. Por otra parte, Estados Unidos ha buscado mantener su posición de liderazgo internacional a través de la regulación de sus actividades y la influencia en las negociaciones internacionales.

Dentro del aspecto económico Estados Unidos se interesó fuertemente por las eventuales actividades económicas que podrían desarrollarse en Antártica. En este sentido, 


\section{A. V. Molina}

este país durante la década de 1980 se pronunció a favor de una explotación de minerales que fuera compatible con la protección del medioambiente, existiendo interés por aprovechar un eventual potencial económico del continente. De esta forma, de acuerdo a Joyner (2013), Estados Unidos tendría principalmente cuatro intereses en la Antártica: “1) mantener la Antártica como una región de cooperación internacional reservada, exclusivamente para fines pacíficos; 2) Preservar y emprender oportunidades únicas para la investigación científica en orden de comprender mejor la geofísica de la Antártica y la tierra y los sistema ambientales; 3) Proteger el relativamente prístino medioambiente de la Antártica y sus ecosistema asociados y; 4) Asegurar la conservación y el manejo sustentable de los recursos vivos en el océano circumpolar del sur (Joyner, 2013, 111). Respecto al desarrollo de investigación científica, Estados Unidos deseaba mantener su posición de liderazgo a través del desarrollo de la ciencia. Como se ha dicho, la ciencia fue utilizada como argumento para alcanzar un consenso para establecer el cogobierno de la Antártica.

Por su parte, en el caso de la normativa internacional se observa que los fines de esta son principalmente científicos, de protección ambiental y de obtención de acuerdos internacionales para el cogobierno de la Antártica. Un punto de encuentro respecto a los fines de ambas normativas es que ambos ordenamientos buscan evitar que la Antártica sea escenario de un conflicto internacional. En este sentido Estados Unidos consideró, al momento de la suscripción del Tratado Antártico, que a pesar de que esta norma sería vinculante para Estados Unidos y limitaría su esfera de acción, este país eventualmente se beneficiaría de la aplicación del derecho internacional a la Antártica porque le permitía evitar controversias en la zona.

\section{Jurisdicción Criminal}

En lo relativo a la jurisdicción criminal sobre crímenes o delitos cometidos en la Antártica la principal diferencia que se observa entre ambos ordenamientos es que Estados Unidos optó por darle una solución general a esta materia, a diferencia de lo que ocurre en el Sistema del Tratado Antártico. Lo anterior porque el Tratado Antártico en el artículo VIII deja el asunto de la jurisdicción sobre personas que realicen actividades en la Antártica sin resolver. Esto dado que la norma establece un régimen de inmunidad de jurisdicción para observadores, científicos y personal auxiliar. De esta forma la situación de las personas que no caben dentro de estas categorías, que el mismo artículo establece, no ha sido resuelta por la normativa internacional, quedando estos grupos de personas sujetos a la jurisdicción de la parte de la cual son nacionales.

En contraste, la preocupación de Estados Unidos por regular esta materia se inició en la década de 1970 motivada, principalmente, por académicos que comprendían que existía un vacío legal en la legislación doméstica y la normativa internacional antártica. En vista de que el Tratado Antártico no solucionaba de forma general el problema de los crímenes 
cometidos en la Antártica por personas no comprendidos en las categorías establecidas en el artículo VIII, luego de varios años de discusión académica y legislativa, se solucionó esta situación con la entrada en vigor de la Comprehensive Crime Control Act el año 1984. Dicha norma a diferencia de la regulación establecida en el Tratado Antártico de 1959, consideró (en concordancia con la política antártica estadunidense) que la Antártica era un lugar que no estaba bajo la jurisdicción de ninguna nación, por lo que este continente era parte de la jurisdicción especial de Estados Unidos. De esta forma las ofensas cometidas por ciudadanos estadounidenses o contra un estadounidense serán de jurisdicción de Estados Unidos

\section{Regulación de Recursos Marinos}

A nivel internacional el primer instrumento que se encargó de la regulación de los recursos marinos antárticos fue la Convención para la Conservación de la Focas Antárticas (1972, en vigor 1978) que prohibía la captura de algunas especies de focas y regulaba la captura de otras. En esta materia se puede concluir que Estados Unidos se adelantó a la regulación internacional, puesto que se adoptó una ley que específicamente regulaba esta materia, la Marine Mammal Protection Act, esta norma propuesta en 1971 al Congreso fue promulgada el 21 de octubre de 1972 y tenía por objeto proteger ciertas especies de mamíferos marinos en peligro de extinción, dentro de los cuales están especies que se encuentran en la Antártica, como las ballenas (Marine Mammal Protection Act of 1972, Pub. L 92-522, 86 Stat.1027, amended through Pub.L109-479, January 12, 2007). Esta iniciativa estadounidense de regular tempranamente los recursos marinos antárticos, se puede explicar porque uno de los intereses de la política internacional antártica de Estados Unidos es asegurar la conservación y un manejo sustentable de los recursos marinos (National Science Foundation, s/f).

Es por esto que funcionarios de la Office of Oceans and International Environmental and Scientific Affairs del Departamento de Estado, la Marine Mammals Commission y la NSF tuvieron un activo rol en la negociación de Convención para la Conservación de Focas. Esta Convención fue negociada en 1972 con el objeto de disuadir a los gobiernos, especialmente la Unión Soviética de autorizar la caza de focas comercial en la Antártica. Como se ha dicho, este instrumento limita protege ciertas especies de focas y limita los métodos, temporadas y áreas para la caza en la Antártica. Asimismo, requiere a las partes que intercambien información y reportes anuales entre ellas y el Scientific Committee on Antarctic Research (SCAR).

Posteriormente, a finales de los 70, Estados Unidos buscó detener el creciente aumento de flotas pesqueras de la Unión Soviética, Japón y Polonia que estaban capturando krill por el impacto que su captura podría generar en otros animales y ecosistemas. De esta forma, Estados Unidos asumió el rol de alcanzar un acuerdo para la entrada en vigor en 1980 de la Convención para la Conservación de los Recursos Vivos Marinos Antárticos (CCRVMA) orientada a conservar y regular la captura de krill y todas las poblaciones antárticas de peces, moluscos, crustáceos y aves marinas que se encuentran al sur del área regulada por 
la Convención de Canberra (1980). Para Estados Unidos, en esos momentos, era crucial asegurar el acceso a pesqueros estadounidenses al océano del sur. Por lo que su mayor preocupación era la necesidad de prevenir y desincentivar la pesca ilegal y no regulada dentro del área (Joyner, 2013, p. 120). A modo de ejemplo, Estados Unidos tuvo una gran influencia en la negociación de la CCRVMA, a través del Antarctic Policy Group y la promoción de la incorporación de ciertas disposiciones claves de la Convención como: la promoción de la creación de un instrumento particular con un enfoque ecológico y sistémico (que considerara el impacto de las actividades humanas y productivas en todo el ecosistema antártico y su efecto nocivo para el medioambiente) y la incorporación de otros estados que no eran parte del Tratado Antártico (Joyner \& Theis, 1997, 106).

A pesar de esta gran influencia, en el caso de la regulación de los recursos vivos marinos la dictación de normativa interna vino después de la adopción de la CCRVMA, en 1984, cuando el Congreso aprobó la Antarctic Marine Living Resources Convention Act. Esto se explica porque era necesaria la entrada en vigor de una ley especial que proveyera la autoridad legal para que Estados Unidos cumpliera sus obligaciones bajo la CCRVMA, por ejemplo: en lo referente a la implementación de las Medidas Conservativas adoptadas por la Comisión de la CCRVMA y la creación de un programa de protección de los recursos marinos, el Antarctic Marine living Resources Program. Es así como, la sección 305(a) (1) de la ley autoriza al secretario de Estado, de Comercio y el director de la NSF, con asesoría del Antarctic Marine living Resources Program a decidir si es que Estados Unidos puede aceptar o no una Medida Conservativa (16 U.S.C. 2434(a) (1).

A modo de síntesis de la comparación de la legislación interna e internacional antártica, en el tópico de recursos naturales marinos, se puede observar que la regulación de Estados Unidos ha estado influenciada fuertemente por un interés en la preservación del medioambiente antártico y la conservación de sus recursos para garantizar una explotación sustentable en el largo plazo y que benefició los intereses estadounidenses en este ámbito. Sin embargo, este interés está estrechamente vinculado con la idea de que, dentro de un marco regulatorio internacional, es su derecho, como estado soberano y de igual modo lo es del resto de los países firmantes del tratado que tengan la capacidad tecnológica, científica y económica, aprovechar los beneficios del desarrollo económico que la Antártica pueda ofrecer.

\section{Regulación de Recursos Minerales}

En un comienzo Estados Unidos promovió enfáticamente la creación de un acuerdo internacional basado en la opinión consensuada internamente de que un acuerdo internacional permitiría solucionar controversias que la futura exploración minera pudiera generar y que permitiría establecer las condiciones bajo las cuales se permitiría el desarrollo de actividades mineras en la zona. El interés de Estados Unidos por los posibles recursos minerales antárticos se remonta a 1939, cuando el Departamento de Estado condujo un estudio sobre la materia. 
Posteriormente, en 1959 un Reporte del National Security Conuncil reconocía que el potencial estratégico y comercial de la Antártica era incierto pero que debía ser considerado, siendo necesaria la elaboración de una normativa "uniforme, no preferencial aplicable a todos los estados para cualquier desarrollo futuro de los recursos antárticos" (Joyner \& Theis, 1997, 136).

De esta forma, la posición de Estados Unidos se ha basado en promover, de ser posible una futura explotación de los recursos minerales, que exista la posibilidad de que los países se beneficien de estas actividades. Asimismo, se ha enfatizado que el desarrollo de la minería se debería dar tomando las debidas consideraciones medioambientales.

Teniendo en cuenta estos principios Estados Unidos promovió activamente el desarrollo de una Convención que regulara las actividades mineras. Así se indicaba en un comunicado de prensa previo a la celebración de la X RCTA en 1979 donde se llamaba a las Partes Consultivas a negociar un régimen minero que considerara las consecuencias medioambientales y al cual Estados Unidos tuviera la oportunidad de compartir "acceso no discriminatorio" a los beneficios, si se comprobaba que las actividades mineras eran factibles de realizar. Este acuerdo se alcanzó con la adopción de la Convención para la Reglamentación de las Actividades sobre Recursos Minerales Antárticos (CRAMRA) en Wellington, 2 de junio de 1988. No obstante, esta Convención no entró en vigor por la falta de apoyo de la comunidad internacional y por la acción de grupos de presión de la sociedad civil estadounidense.

En este contexto, y como estrategia alternativa, el Congreso estadounidense promovió posteriormente el abandono de la CRAMRA y la creación de un acuerdo internacional que prohibiera las actividades sobre recursos naturales en la Antártica. Por esta razón, y por la posibilidad de perder el liderazgo en la Antártica, el Ejecutivo optó por abandonar la CRAMRA y promover la creación de una legislación doméstica que prohibiera las actividades mineras. Este objetivo se cumplió con la aprobación de la Antarctic Conservation Act de 1990, donde además de prohibirse las actividades mineras se llamaba al gobierno a alcanzar una solución internacional para la protección de la Antártica. Al año siguiente se adoptaría el Protocolo de Madrid.

Una conclusión preliminar de este tópico de comparación, es que Estados Unidos ha mostrado interés en la investigación y evaluación de los posibles beneficios económicos que pudieran existir en la Antártica, de su factibilidad económica y de las posibilidades que la normativa internacional les otorga de tener acceso a desarrollar este tipo de actividades. A pesar de la fuerte influencia y presión que ejerció Estados Unidos para el desarrollo de un régimen minero el rechazo de la comunidad internacional y nacional le impidió consolidar uno de sus objetivos geopolíticos. De esta forma con la dictación de la Antarctic Protection Act de 1990 se evidencia una concesión del gobierno de Bush al Congreso y los grupos ambientalistas, motivada principalmente por la necesidad de mantener la estabilidad del Sistema del Tratado Antártico. En este sentido, habría existido un "principio de discordancia" entre lo dispuesto en la legislación internacional y la posición de Estados Unidos sobre las actividades mineras, 


\section{A. V. Molina}

situación que no llegó a concretarse, por el cambio de enfoque en esta materia que llevaría a impulsar la creación de una nueva regulación ambiental para la Antártica.

\section{Protección al Medioambiente}

En lo referido a la protección medioambiental, la conservación y protección de la Antártica ha sido uno de los intereses de Estados Unidos. En este sentido, el primer instrumento adoptado a nivel internacional fueron las Medidas Acordadas para la Conservación de la Flora y Fauna en 1964. Estados Unidos incorporó dicha normativa internacional en su derecho doméstico a través de la aprobación de la Antarctic Conservation Act de 1978 (Browne y Mielke, 1995).A pesar de que dicha norma aseguraba la protección de la flora y la fauna, la protección del ecosistema y el desarrollo de una regulación el control de la contaminación, fue criticada por grupos ambientalistas que señalaban que la NSF no habría cumplido la norma, específicamente en cuanto a la lenta implementación de las normas sobre polución.

Posteriormente, en 1990 se puede observar una fuerte influencia de Estados Unidos en la promoción de la negociación del Protocolo Medioambiental. Esto se demuestra en que durante las discusiones en el Congreso sobre la ratificación de la CRAMRA se inició una fuerte presión no solo para abandonar este acuerdo sino que además para llamar a la celebración de un nuevo acuerdo internacional que promoviera la protección del medioambiente. Es así que en la Antarctic Protection Act de 1990 se llamaba al gobierno a alcanzar un nuevo acuerdo internacional que tuviera por objeto la protección del medioambiente antártico. En la implementación de esta iniciativa legal se puede observar que Estados Unidos con el fin de mantener su liderazgo en materia antártica, cambió la posición sostenida en la década de los 80 que promovía las actividades mineras y decidió tener un rol activo en las negociaciones del Protocolo Medioambiental. En 1989 Estados Unidos, Chile, Nueva Zelanda y Suecia presentaron un borrador de propuesta de medidas de protección en la RCTA de París. El resultado fue la realización de una serie de reuniones especiales que iniciaron con una RCETA en Viña del Mar en diciembre de 1990 y que terminaría con la suscripción del Protocolo Medioambiental el año 1991 en Madrid.

\section{CONCLUSIONES}

En términos generales se puede sostener que la normativa interna generada en Estados Unidos en relación con la Antártica ha tenido una creciente influencia en el surgimiento y evolución del Sistema del Tratado Antártico. Una prueba de ello es que Estados Unidos influyó sustancialmente en la materialización de un acuerdo internacional para la adopción del Tratado Antártico, logrando con ello que sus intereses nacionales de carácter geopolíticos se vieran reflejados en este instrumento. Asimismo, se observa que este país, por su creciente carácter de potencia mundial, ha tenido un rol relevante en el desarrollo de los principales 
instrumentos internacionales que regulan la Antártica. Es así como, en la actualidad Estados Unidos ha logrado consolidar y mantener tanto su posición de liderazgo político, así como fuente de financiamiento y actor relevante en materia de logística antártica que promueve el desarrollo de investigaciones científicas de largo plazo y con presencia humana permanente en el continente antártico. Lo anterior, es complementado con una fuerte actividad diplomática y presencia permanente en todos los foros y organismos antárticos internacionales.

Por otra parte, se puede concluir que en Estados Unidos existe una normativa orgánica encargada de regular las actividades desarrolladas por este país en la Antártica. El proceso de creación de este régimen jurídico se inició con posterioridad a la entrada en vigor del Tratado Antártico en 1961 y su desarrollo se encuentra estrechamente vinculado con las negociaciones de acuerdos internacionales en el marco del Tratado Antártico. Como se ha dicho anteriormente las negociaciones del Tratado, estuvieron influenciadas por las directrices de la política antártica desarrollada por el Departamento de Estado desde inicios del siglo veinte. Desde mediados de este siglo Estados Unidos intensificó su interés por la Antártica, desarrollando una serie de expediciones que buscaban afianzar su posición en la zona y consolidar su área de influencia en diversos ámbitos. Del análisis realizado se observa que este interés de Estados Unidos estuvo motivado por razones geopolíticas, estratégicas y económicas, más que por razones científicas o conservacionistas.

En relación a la comparación entre la interacción entre normativa internacional y la normativa interna de Estados Unidos, se observó que la normativa internacional antártica se encuentra fuertemente influenciada por el rol que ha tenido Estados Unidos en el Sistema del Tratado Antártico, lo que se observa en las negociaciones de la Convención de Londres, el Protocolo Medioambiental, la Convención para la Reglamentación de las Actividades sobre Recursos Minerales Antárticos y la Convención sobre Conservación de los Recursos Vivos Marinos Antárticos. A su vez se observa que Estados Unidos en el caso específico de la normativa internacional antártica ha implementado los acuerdos internacionales suscritos. Esta situación se aparta de la doctrina que argumenta que la adopción de acuerdos internacionales por parte de Estados Unidos no ha ido acompañada de una consolidación en el ordenamiento interno, sosteniéndose la idea de independencia de las instancias nacionales respecto de las obligaciones emanadas del derecho internacional.

En definitiva se concluye que existe una influencia mutua del derecho internacional sobre el derecho interno, y de Estados Unidos sobre el desarrollo de los instrumentos que componen el Sistema del Tratado Antártico. De esta forma, la realización de este análisis comparativo, nos permite comprobar la existencia de una correlación entre el desarrollo la normativa antártica interna estadounidense y el desarrollo del régimen jurídico internacional que rige a la Antártica. Estados Unidos ha utilizado su influencia como potencia mundial para influenciar el logro de acuerdos dentro de los países que tienen intereses antárticos y para fomentar las ideas que sostienen su política antártica, que buscan la satisfacción de sus intereses nacionales y geopolíticos. 
Es así como, Estado Unidos con la promoción y participación activa que ha tenido en el desarrollo de la normativa internacional antártica, ha llevado a que este país desarrollara y consolidara una completa normativa doméstica que orienta de modo específico y claro las actividades de personas naturales e instituciones americanas en el continente antártico. En definitiva, la presente investigación nos permite concluir que, actualmente, en el ordenamiento jurídico interno de Estados Unidos existe una normativa específica que regula las actividades que nacionales e instituciones realizan en el continente y que dicha normativa se expresa en un conjunto de leyes y normativas administrativas que constituyen un completo marco legal.

La determinación de que Estados Unidos cuenta con una abundante regulación de sus actividades antárticas, que abarca distintas dimensiones de estas actividades, resulta de gran interés por cuanto Estados Unidos, no tiene reclamaciones de soberanía sobre la Antártica y desconoce que los Países Reclamantes ejerzan algún tipo de jurisdicción sobre ella. Por el contrario, a pesar de esta política antártica, Estados Unidos desde el año 1950 ha tenido una activa presencia en la zona, que se evidencia, no solo, en el número de bases, investigaciones científicas desarrolladas, y las actividades pesqueras y de exploración que desarrolla, sino que también a través de la creación de este completo marco jurídico para la regulación de sus actividades en la Antártica.

A pesar de la importancia que tiene Estados Unidos en el Sistema del Tratado Antártico, en la actualidad han surgidos críticas por la falta de inversión de este país en infraestructura y logística para la investigación antártica. En este sentido, la NSF ha sugerido que en orden de asegurar la capacidad de investigación, la presencia en el polo sur y los intereses de Estados Unidos en la Antártica, se hace imprescindible una modernización de la capacidad de rompehielos operativa en el territorio antártico. En este contexto, otras potencias mundiales han emergido fuerte y rápidamente, este es el caso de China, quien ha demostrado un creciente interés sobre la Antártica, colocándola como una de sus prioridades nacionales estratégicas. Esta situación podría estar evidenciando que la posición de liderazgo que Estados Unidos desarrolló en el curso del siglo veinte se estaría viendo amenazada si es que el gobierno estadounidense no coloca como prioridad a la Antártica como lo hizo casi 60 años atrás.

\section{REFERENCIAS}

Abdel-Motaal, D. (2016). Antarctica: the battle for the seventh continent. Santa Barbara, CA: Praeger.

Aguayo, A. (2005). El año geofísico internacional y su importancia para el desarrollo de la ciencia antártica chilena. En M. C. León Wöppke, M. Jara \& J. Kendell (Eds.), ¿La Convergencia Antártica?: Los Contextos de la historia antártica chilena 1939-1949, (pp. 31-37). Valparaíso: Puntángeles Universidad de Playa Ancha.

Bastmeijer, K. (2000). Implementing the Environmental Protocol Domestically: An Overview. En Davor Vidas (Ed.), Implementing the Environmental Protection Regime for the Antarctic. 
Environment \& Policy (pp. 287-307). Dordrecht, Netherlands: Springer Netherlands. Berguño, J. (2009). El Tratado Antártico como Régimen Internacional, Diplomacia, 120, 22-34. Browne, M. y Mielke, J. (1995). Antarctica:Environmental Protection, Research, and Conservation of Resources. National Library for the Environment. Recuperado de https://digital. library.unt.edu/ark:/67531/metacrs192/m1/1/high_res_d/95-476_1995Apr05.html Buedler, W. (1957) El Año Geofísico Internacional. La UNESCO y su programa, UNESCO. Recuperado de http://unesdoc.unesco.org/images/0012/001283/128396so.pdf Day, D. (2012). Antarctica a biography. Reino Unido: Oxford University Press.

Díaz, R. y Villamizar, F. (2014). Uso Pacífico de la Antártica como Norma de Ius Cogens. Magallania, 1, 17-31.

Dodds, K. (2009). La Administración del Continente Polar: Los Orígenes Geopolíticos del Tratado Antártico de 1959". Istor, 39, 27-49.

Ferrada Walker, L. (2012). Evolución del Sistema del Tratado Antártico: Desde su génesis geoestratégica a sus preocupaciones ambientalistas. Revista de Derecho Universidad San Sebastián Chile, 18, 131-151.

Ferrada Walker, L. (2014), Iniciativa privada y estatal en la actividad antártica. Pasado, presente y futuro. En XLIII Jornadas chilenas de derecho público: Estado, sociedad y participación, Universidad de Concepción/Thomson Reuter/LegalPublishing, 1-16.

Ferrada Walker, L. (2015). La Antártica ante la Corte Internacional de Justicia: A 60 años de los casos Reino Unido c. Chile y Reino Unido c. Argentina, Tribuna Internacional, 7, 155-172.

Hall, R. 1989. The "open door" into Antarctica: an explanation of the Hughes doctrine. Polar Record, 25, 137-140.

Ireland-Piper, D. (2017). Accountability in extraterritoriality: A comparative and international law perspective. Northampton, MA: Edward Elgar.

Jara, M. (2009). Chile y Estados Unidos en la Antártica 1923-1939: De la V Conferencia panamericana al inicio de la Segunda Guerra Mundial. Estudios Norteamericanos, 19-20, 105-125.

Jara, M., \& Mancilla, P. (2016). Chile y Estados Unidos: Intereses y Políticas en la Antártica 1928193. Historia, 396, 313-339.

Jara, M. (2005). Richard Evelyn Byrd y Ramón Cañas Montalva: Contrapunto de visiones antárticas hacia 1940. En M. C. León Wöppke, M. Jara \& J. Kendell (Eds.), ¿La Convergencia Antártica?: Los Contextos de la historia antártica chilena 1939-1949 (pp. 151-159). Valparaíso: Puntángeles Universidad de Playa Ancha.

Joyner, C., \& Theis Ethel. R. (1997). Eagle over the ice. The U.S. in the Antarctic. Estados Unidos: University Press of New England.

Joyner, C. (2013). United States foreign policy interests in the Antarctic, En A. M. Brady (Ed.), The Emerging Politics of Antarctica, (pp. 110-129), Hamburg: Routledge.

Lackenbauer, W., \& Kikkert, P. (2015). The Dog in the Manger and Letting Sleeping Dogs Lie: 
The United States, Canada and the Sector Principle, 1924-1955. En S. Lalonde, \& T. McDorman (Ed.), International Law and Politics of the Arctic Ocean Essays in Honor of Donat Pharand (pp. 216-239). Leiden: Brill.

León, M. C. (2005). El conocimiento antártico estadounidense a mediados de la decada de 1930: El aporte de la American Philosophical Society. En M. C. León, M. Jara \& J. Kendell (Eds.), ¿La Convergencia Antártica?: Los Contextos de la historia antártica chilena 19391949 (pp. 17-30). Valparaíso: Puntángeles Universidad de Playa Ancha.

León, M. C. (2005). La Cuestión Antártica, 1939-1941: ¿Una Disputa Prefabricada?. En M. C. León Wöppke, M. Jara \& J. Kendell (Eds.), ¿La Convergencia Antártica?: Los Contextos de la historia antártica chilena 1939-1949 (pp. 61-74). Valparaíso: Puntángeles Universidad de Playa Ancha.

León, M. C. (2005). Política y Políticas Antárticas Estadounidenses en la Década de 1940. En M. C. León, M. Jara \& J. Kendell (Eds.), ¿La Convergencia Antártica?: Los Contextos de la historia antártica chilena 1939-1949 (pp. 31-41). Valparaíso: Puntángeles Universidad de Playa Ancha.

Llanos, N. (2008). La “Troya Antártica”: Estados Unidos, guerra fría y ciencia en el continente helado, 1948-1959. Estudios Norteamericanos, 18, 177-225.

Mancilla, P. (2004). El proyecto de Internacionalización Estadounidense de la Antártica y la Reacción del Ministerio de Relaciones Exteriores de Chile en 1948. Estudios Norteamericanos, 3, 157-163.

Mancilla, P. (2005). La operación estadounidense “Deep Freeze I” en la Antártica vista por la Estrella de Valparaiso, 1955-1956. Estudios Norteamericanos, 12, 83-90.

Mancilla, P. (2006). Chile, Argentina y Gran Bretaña en el Continente Antártico, 19061961. Una Aproximación a las Controversias Diplomáticas, Revista de Estudios Históricos, 3. Recuperado de http://www.estudioshistoricos.uchile.cl/CDA/est_hist_ impresion/0,1476,SCID\%253D18825\%2526ISID\%253D650,00.html.

Moore, J. (2005). A Typical Marriage: U.S.-British-Chilean Antarctic Relations, 1946-1949. En M. C. León Wöppke, et al. ¿La Convergencia Antártica?: Los Contextos de la historia antártica chilena 1939-1949 (pp. 193-210). Valparaíso: Puntángeles Universidad de Playa Ancha.

Orrego Vicuña, F. (1984). Derecho internacional de la Antártida. Chile: Dolmen Ediciones. Pinochet de la Barra, O. (1976). La Antártica Chilena. Santiago: Editorial Andrés Bello.

Plott, B. (1969). The Development of United States Antarctic Policy. Tesis Doctoral, Tufts University.

Saul, B., \& Stephens, T. (2015). Antarctica in international law. Oxford: Hart Publishing.

Trius, L. (2016). La precariedad del derecho internacional en los Estados Unidos: Los Casos Breard y Lágrand. Revista Jurídica Universidad Autónoma de Madrid, 2 (200), 177-214.

United States Antarctic Program, U.S. Antarctic Program Participant Guide, 2014-2016, United States Antarctic Program, [online]. Recuperado de https://www.usap.gov/ 
travelAndDeployment/documents/ParticipantGuide-Chapter1.pdf.

Villamizar, F., Ibarra, P., \& Guerrero, C. (2013). Territorio Antártico Chileno: La Reacción De

Chile Ante Los Intereses Antárticos Globales, Revista de Relaciones Internacionales, Estrategia y Seguridad, 2, 95-116.

\section{NORMAS DE ESTADOS UNIDOS E INTERNACIONALES CONSULTADAS}

Antarctic Conservation Act of 1978. Pub. L. 95-541, 92 Stat. 2048, 16 U.S.C. §§ 2401 et seq. Antarctic Marine Living Resources Convention Act of 1984, Public Law 98-623, 98 Stat. 3398, 16 U.S.C. §§ 2431 et seq.

Antarctic Treaty System, "Informe Final de la Cuarta Reunión Consultiva Especial del Tratado Antártico Sobre Recursos Minerales Antárticos”, Antarctic Treaty System, Recuperado de https://www.ats.aq/documents/SATCM4_12/fr/SATCM4_12_fr001_s.pdf.

Comprehensive Crime Control Act of 1984, Public Law 98-473, 3 U.S.C, § 1210, (1984), 98 Stat. 2164.

Convención para la Conservación de las Focas Antárticas, Londres, 1988.

Convención para la Conservación de los Recursos Vivos Marinos Antárticos, Canberra, 1980. Convención para la Reglamentación de las Actividades sobre Recursos Minerales Antárticos, (CRAMRA), Wellington, 2 de junio de 1988.

Illegal, Unreported, and Unregulated Fishing Enforcement Act de 2015, Public Law 114-81. Marine Mammal Protection Act of 1972, Public Law 92-522, 86 Stat.1027, amended through Pub.L109-479, January 12, 2007.

Protocolo al Tratado Antártico sobre la Protección del Medio Ambiente, Madrid, 1991.

Tratado Antártico, 1 de diciembre de 1959, Washington, DC, Estados Unidos 
